\title{
Mujeres con discapacidad y asistencia personal: la experiencia de Aspaym Málaga
}

\author{
Women with disabilities and personal assistance: the experience of \\ Aspaym Málaga
}

\section{Palabras clave}

Mujeres, dependencia, empoderamiento, asistencia personal, autonomía.

\section{Keywords}

Women, dependence, empowerment, personal assistance, autonomy.

\section{Isabel García Trigueros \\ <isagatri@gmail.com>}

Aspaym Málaga. España

1. Introducción. La perspectiva de género del derecho a una vida independiente

En este artículo se analiza la experiencia de una entidad como Aspaym Málaga, que contribuye a la igualdad mediante el trabajo de un equipo multidisciplinar en un ámbito poco conocido aún en el mundo asociativo y político de nuestro país como es la asistencia personal. Su objetivo es promover la inclusión y la participación de las mujeres en una vida socialmente activa y para ello se desarrollarán acciones en tres fases.

La primera, de autonomía e independencia, comprenderá el estudio de las situaciones personales y de empoderamiento que las llevó a decidir emprender un nuevo camino - a pesar de las discriminaciones derivadas de la discapacidad y su perspectiva - teniendo en cuenta los derechos humanos y cómo las mujeres con discapacidad son capaces de recuperar su vida social y de empoderarse cuando salen del entorno familiar.

La segunda prestará atención a la asistencia personal, formación y medio. Se observará el compromiso de las entidades de mujeres con discapacidad, la adaptación de un equipo multidisciplinar que arroje luz al compromiso de las entidades ante la realidad discriminatoria de las mujeres desde una visión positiva de la discapacidad, por un lado mediante la formación

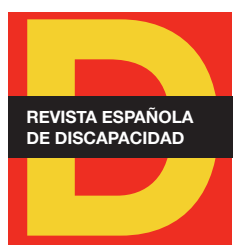

Para citar:

García, I. (2020). "Mujeres con discapacidad y asistencia personal: la experiencia de Aspaym Málaga". Revista Española de Discapacidad, 8(2), pp. 247-254.

Doi: <https://doi.org/10.5569/23405104.08.02.13> 
y empoderamiento de las mujeres $y$, por otro lado, realizando actividades formativas para las personas que van a colaborar en dar la máxima autonomía, lejos de un sistema básico exclusivamente de cuidados.

Y la tercera fase de este artículo es el resultado conseguido después de cuatro años de proyecto y en la que se presentarán tres ejemplos de mujeres con discapacidad y su inclusión en la sociedad. Por último, se incluye una conclusión derivada de las dificultades de las entidades y de las mujeres con discapacidad para acceder a este medio de inclusión, en este caso en el ámbito de Andalucía, y un debate a futuro sobre la regulación legal de este instrumento.

La convención de la ONU sobre derechos de las personas con discapacidad en su artículo 19 ratifica el "Derecho a vivir de forma independiente y a ser incluido en la comunidad".

Por su parte, la Constitución Española establece el derecho a igualdad ante la ley en su artículo 14, y uno de los principios rectores de la política social, en el artículo 49, reconoce que los poderes públicos realizarán políticas públicas para el disfrute de los derechos de las personas con discapacidad.

La aprobación de la Ley 39/2006, de 14 de diciembre de Promoción de la Autonomía Personal y Atención a las personas en Situación de Dependencia (LAPAD), en su artículo 19 sobre prestación económica de asistencia personal establece: "La prestación económica de asistencia personal tiene como finalidad la promoción de la autonomía de las personas en situación de dependencia en cualquiera de sus grados".

En el ámbito territorial de la Comunidad Autónoma de Andalucía, el I Plan Andaluz de la promoción de la autonomía personal y prevención de la dependencia 2016-2020 define la asistencia personal y su prestación económica.

El rol de género en las políticas sociales destinadas a las personas con discapacidad es prácticamente inexistente, aunque desde la filosofía de vida independiente y los colectivos de mujeres con discapacidad analizamos que la perspectiva de género es un plus añadido a la multidiscriminación de las mujeres y un límite a la inclusión en la comunidad.

Hay casi dos millones y medio de mujeres con discapacidad en España, lo que supone un $60 \%$ de las personas con discapacidad de todo el Estado, es por ello que la perspectiva de género se hace imprescindible. Desde Aspaym Málaga, con un equipo multidisciplinar formado por la junta directiva, la trabajadora social y la terapeuta ocupacional, detectamos los factores que limitaban a las mujeres con dependencia para realizar las actividades de la vida diaria y el compromiso de la sociedad con ellas, como son los personales, familiares y sociales derivados del sistema patriarcal que las minimiza bajo formas de normalización de las situaciones de violencia institucional, social, familiar y comunitaria que las impiden ser sujetos de pleno derecho (Martín y Ruiz, 2010).

Después de detectar estas carencias, se estableció una estrategia de mejora de la calidad de la vida pública de las mujeres con discapacidad y dependencia de la asociación y que, posteriormente derivó, hacia un acercamiento de otras mujeres de diferentes asociaciones y ámbitos, tanto de los cuidados como parejas de hombres y mujeres con discapacidad, etc. 


\section{Método. Las fases hacia una vida independiente sin barreras a la discriminación por razón de género (Fases 1 y 2)}

Las mujeres con discapacidad se enfrentan a situaciones de discriminación múltiple tanto por género como por su propia discapacidad (CERMI, 2018: 255-266). Existe una violencia estructural derivada del patriarcado que, en el caso de las mujeres con grandes dependencias, se manifiesta en la institucionalización forzosa (Huete, 2015), la infantilización y el sentimiento de carga que suponen para las mujeres de su familia - siempre ellas las cuidadoras-, lo que las impide llevar una vida autónoma y las desprovee de tomar de decisiones. Las consecuencias de todo ello son la baja autoestima, vulnerabilidad y discriminación, con el añadido de no poder asumir, como mujeres, las tareas que el sistema les asigna.

Para establecer los objetivos teniendo en cuenta estos parámetros, decidimos comenzar realizando unas acciones positivas destinadas a prevenir la baja autoestima y a facilitar el empoderamiento de las mujeres con gran dependencia. Para ello, consideramos la educación en el feminismo como referente para evitar desigualdades derivadas del patriarcado y desarrollamos teorías como "el escalón de cristal" (González, 2010) para salvar las distancias con otras discriminaciones o desigualdades.

\subsection{Fase 1. El empoderamiento de las mujeres con discapacidad}

La definición de empoderamiento para mujeres con discapacidad física y dependencia tiene una connotación específica y una definición propia: se entiende como la posibilidad de las mujeres de tener autodeterminación y control de la toma de decisiones sobre su propia persona a través de medios o técnicas de desarrollo de la personalidad. El taller "Ponte guapa, ponme guapa" - nombre derivado de la importancia de necesitar otra persona como medio para el empoderamiento - se desarrolla diariamente durante tres meses con el objetivo específico de conseguir que las mujeres tomen conciencia de su propio cuerpo. En ese tiempo se potencia su autoestima por medio de la relajación y la psicoterapia y que así logren ser conscientes de que tienen capacidad plena para tomar decisiones que afecten a su propia vida social, familiar y en comunidad.

En las jornadas de activismo para mujeres con discapacidad se pasa al nivel de toma de conciencia del entorno, las discriminaciones múltiples que sufren, la visión paternalista y patriarcal de la sociedad ante las mujeres con discapacidad. Para ello, las asistentes realizan una puesta en común sobre estas situaciones y desarrollan posibles soluciones. Se forma a las mujeres sobre feminismo y el movimiento feminista de la discapacidad, el discafeminismo, para que sean conscientes de la teoría del "escalón de cristal" y tengan los medios para poder saltarlo, cuestión que nos lleva a otra problemática de la que se tratará en las conclusiones.

\subsection{Fase 2. El camino hacia la autodeterminación, la formación es el medio}

El sistema de cuidados y su gestión es fundamental en situaciones de dependencia y empoderamiento de mujeres con discapacidad y gran dependencia. El 58 \% de las personas en situación de dependencia son 
mujeres y sus cuidadoras son también mujeres; la mayor parte pertenecen al ámbito familiar (madres, abuelas, hermanas) que abandonan o no entran en el mercado laboral activo para poder realizar dicho cuidado. Desde Aspaym Málaga se observa esta situación como limitación al derecho subjetivo y político de ambos grupos de mujeres, cuidadoras/familiares y dependientes, a tener una vida socialmente activa y una barrera a la consecución del proyecto, que se verá más adelante.

Por ello, la intervención tiene una doble dimensión. Por un lado se incluyó a las mujeres cuidadoras/familiares en los talleres de empoderamiento y, por otro, se inició un proyecto de formación de asistentes personales para personas con discapacidad y dependencia.

La asistencia personal está dentro de la Declaración Universal de los Derechos Humanos (Foro de Vida independiente y Divertad, 2015). Tomando como referencia la filosofía de vida independiente, esta se consideró como el medio, es decir, la ayuda mutua de una persona que ayuda a otra a desarrollar su vida de forma que tenga autodeterminación suficiente para ejercer su vida en igualdad de oportunidades y, en el caso de las mujeres, tal como se planteó en Aspaym Málaga, para poder superar la teoría del "escalón de cristal".

En estas jornadas formativas que se desarrollan durante cuatro días se estudian aspectos básicos, definiciones y legislación sobre la asistencia personal con módulos específicos sobre el impacto de género en la dependencia, la necesidad de concienciación de las personas profesionales ante estas situaciones de multidiscriminación, la interacción de estas personas con la sociedad, la empatía, la gestión de conflictos a la hora de la toma de decisiones o el respeto a la intimidad personal, además de otros aspectos sociosanitarios.

\subsection{Resultado. Fase 3. Las mujeres en primera persona}

Con el objetivo general cumplido y los específicos realizados durante cuatro años desde el equipo multidisciplinar de Aspaym Málaga, se deciden publicar las conclusiones presentando en este artículo a tres mujeres jóvenes con discapacidad y gran dependencia que, una vez realizadas las fases 1 y 2 , llevan una vida prácticamente autónoma gracias a su propio esfuerzo, el de sus asistentes personales y el apoyo de la entidad. 
Imagen 1. Foto de Nerea

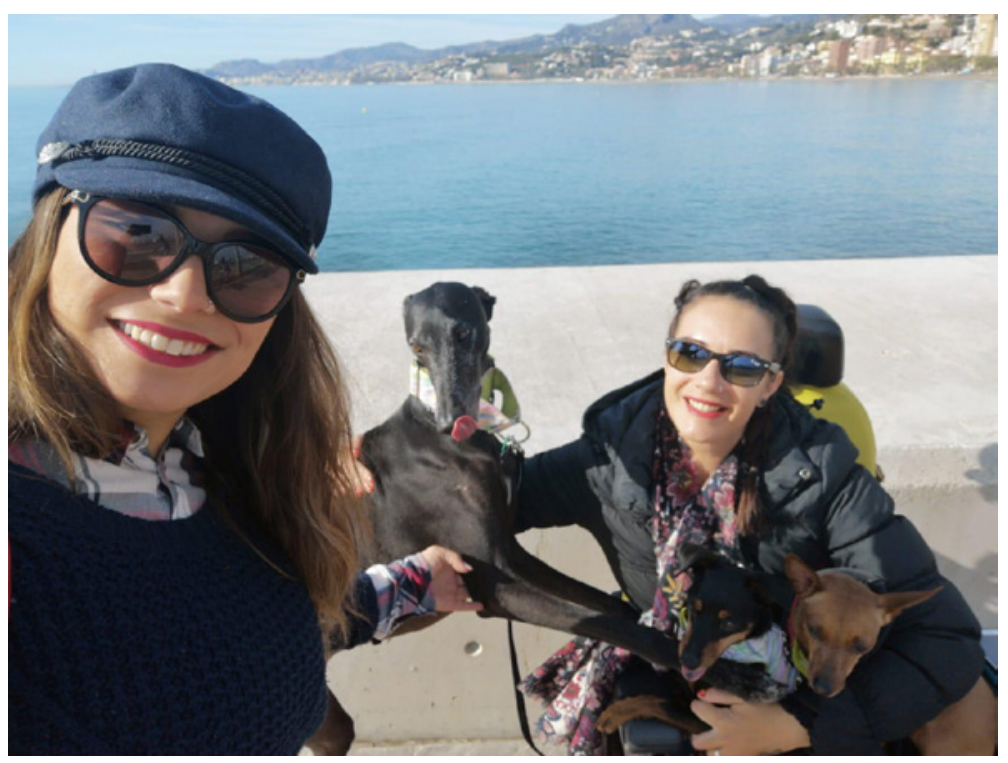

Imagen 2. Foto de Melania

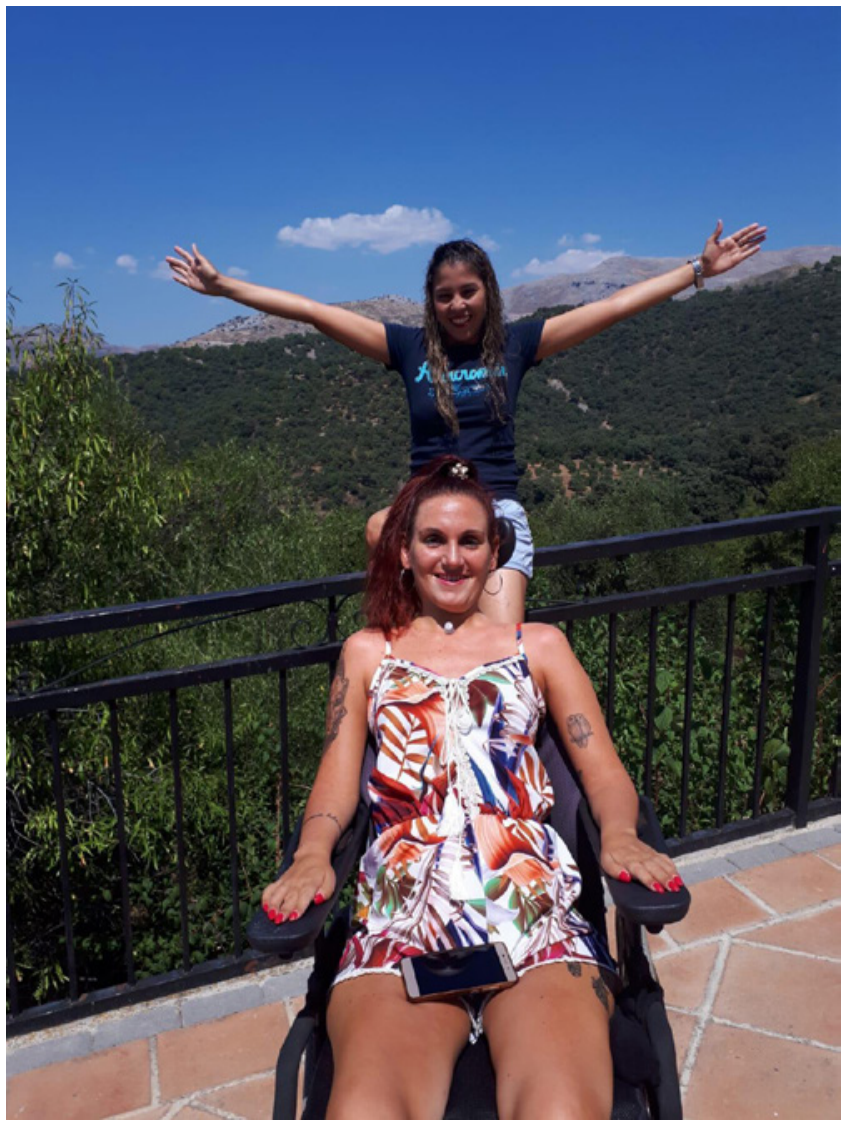




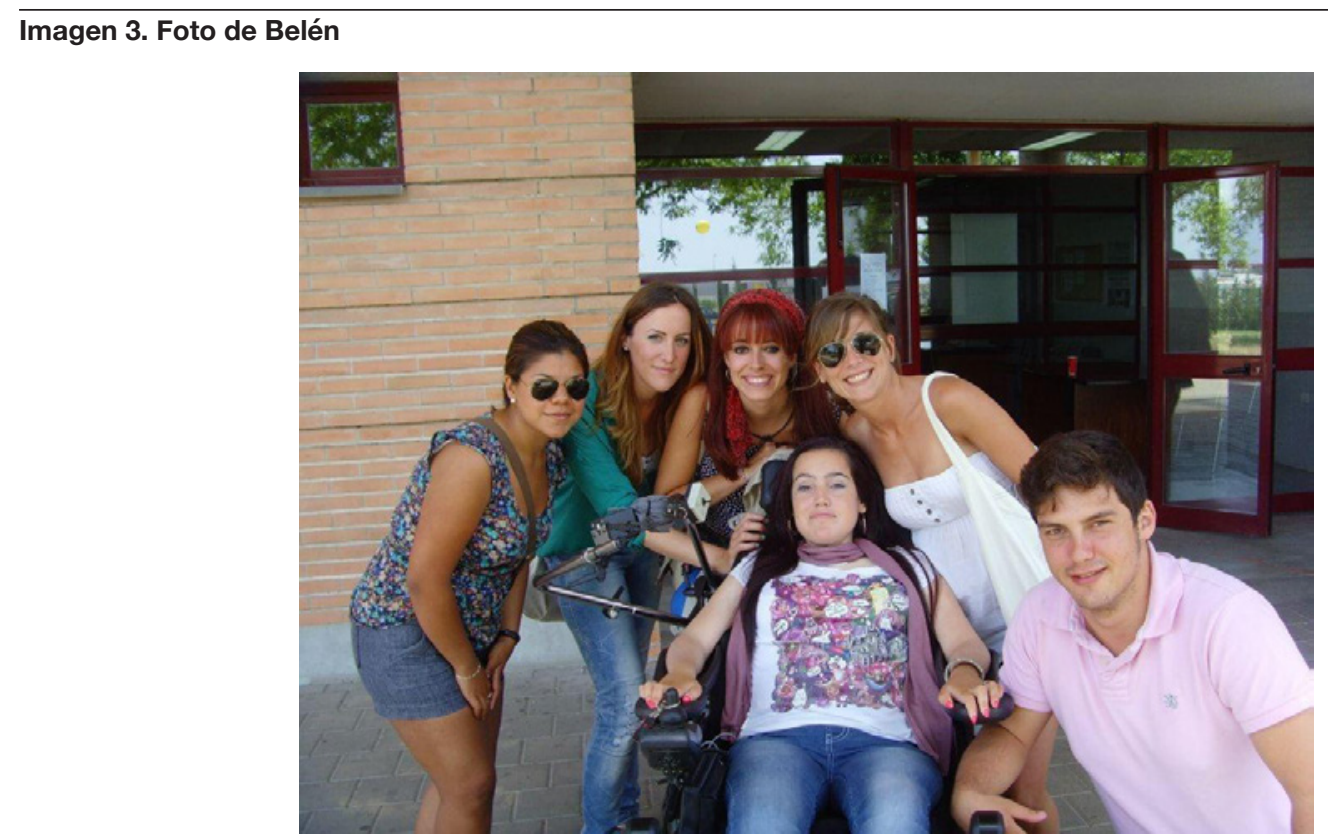

\section{Conclusión y discusión}

Desde Aspaym Málaga se ha hecho un balance de la situación de veinte mujeres que mejoraron notablemente su situación. Los aspectos positivos fueron la mejora de su autoestima y de su relación familiar. La toma de decisiones es mayor cuanta más confianza tienen con su nueva vida social activa, aumentan los ámbitos de participación de la vida pública, son sujetos de concienciación de otras personas dependientes como ejemplo para ellas, se autorreconocen como sujeto de derechos y la autodeterminación forma parte de su vida diaria, algo que se consideró muy importante por la perspectiva negativa que vamos a tratar en seguida.

Por otro lado se han determinado tres aspectos negativos específicos desde la perspectiva de las entidades y con una visión feminista de la diversidad:

- Primero, tratar de saltar el "escalón de cristal", parece que solo compete a las mujeres y a las entidades de mujeres con discapacidad (según ONG, las entidades del tercer sector están dirigidas solo por un $28 \%$ de mujeres). La perspectiva y la teoría de género es prácticamente inexistente en las políticas activas por tanto tendemos a "responsabilizar" de alguna manera, echar más carga a las mujeres para que salgan y se empoderen mientras que la sociedad, la política y el entorno no tienen interés alguno en prepararse para la verdadera inclusión y por tanto estamos ante una "integración impuesta" y no ante una verdadera inclusión. 
- Segundo, la legislación sobre dependencia no contempla suficientemente ni a las mujeres en situación de dependencia, ni aspectos sobre multidiscriminación o violencia. La atención es básicamente asistencialista y no empoderante en general, sin especificar ámbitos sobre impacto de género o consecuencia en la situación de cuidadoras/familiares, o de asistencia personal.

- Tercero, el acceso a la asistencia personal por parte de estas mujeres se puede hacer a través de tres medios fundamentalmente, que son:

1. Ley de dependencia directa (insuficiente y mediante un largo proceso de justificaciones) de entidades (por proyectos que apenas tiene recursos contemplados en leyes de presupuestos).

2. Contratación de servicios por entidades, lo que lleva a un coste excesivo.

3. Contratación directa, lo que lleva a que el asistente personal tenga que costear su propia cuota de la Seguridad Social como empleada de hogar, lo que implica además precariedad laboral.

Se necesita más investigación, más conclusiones, mayor implicación y coordinación de políticas activas y transversales de inclusión que deje a un lado la visión paternalista y patriarcal para dar paso a políticas feministas de inclusión específicas para mujeres con dependencia. 


\section{Referencias bibliográficas}

Comité Español de Representantes de Personas con Discapacidad, CERMI (2018). Derechos Humanos y Discapacidad. Informe España 2017. Madrid: Ediciones CINCA. Recuperado de https://bit.ly/37rahhs.

Foro de Vida independiente y Divertad (2015). La Asistencia Personal como Derecho Humano. Propuesta política. Recuperado de https://bit.ly/39KQEn8.

González, C. (2020). El escalón de cristal. Discafeminismo. Claves para comprender la discriminación múltiple. Granada: Editorial Dauro.

Huete, A. (dir.) (2015). Institucionalización de las personas con discapacidad en España. Madrid: Observatorio Estatal de la Discapacidad. Recuperado de https://bit.ly/2KSSGHx.

Martín, M. C. y Ruiz, S. (2010). "Ley de Dependencia: una mirada con perspectiva de género". Documentos de Trabajo Social, 48, pp. 84-97. 